\title{
Regional comparison and countermeasure research of Chinese herbal medicine planting willingness in Shandong Province
}

\author{
Xin $\mathrm{Su}^{1, \mathrm{a}}$, Wenzhe $\mathrm{Hu}^{2, \mathrm{~b}}$, Renzhu $\mathrm{Yu}^{2,{ }^{*}}$ \\ ${ }^{1}$ School of Business Administration Shandong University of Finance and Economic \\ No.7366, East Erhuan Road, Jinan, 250014 China \\ ${ }^{2}$ School of Business Administration Shandong University of Finance and Economic \\ No.7366, East Erhuan Road, Jinan, 250014 China \\ * feihu_yu@163.com,* \\ ( ${ }^{*}$ means corresponding author)
}

Keywords: Chinese herbal medicine, Planting will, regional comparison

\begin{abstract}
Chinese herbal medicine is a treasure of historical and cultural traditions of the Chinese nation, also is an important carrier of the development of Chinese medicine culture communication, and the cornerstone of the development of medical and health in China. The protection and development of Chinese herbal medicine industry is of great significance of improving the medical level in our country, improving Chinese people's life quality and developing new industries. The research focus of this paper is the difference of Chinese herbal medicine planting area in Shandong Province, which is of great significance to solve the problems in the development of Chinese herbal medicine industry in the future. This thesis is divided into five parts, The first part is the introduction, the second part is the present situation of herbal medicine planting in Shandong Province, the third part is the comparison of Chinese herbal medicine planting areas in Shandong Province, the forth part is the problems in the industrial development of Chinese herbal medicine planting in Shandong Province, the last part is countermeasures of developing Chinese herbal medicine planting industry in Shandong Province.
\end{abstract}

\section{Introduction}

With the development and progress of society, the consumption concept of "respect for nature and return to nature" is more popular, people prefer green natural medicines and health care products, and Chinese herbal medicine market demand has become increasingly strong. Especially with the recent announcement of the 2015 Nobel Prize in medicine, Chinese herbal medicine value has been recognized around the world. It provides more opportunities for the development of traditional Chinese medicine industry, and the prospects for the future development of Chinese herbal medicine industry in our country are good.

Based on the current understanding of the development of Chinese herbal medicine industry in Shandong Province, the report took the regional differences in Chinese herbal medicine industry in Shandong Province as the breakthrough point, investigated and summarized the index of planting area in major producing areas, planting area distribution, main varieties, total output, processing capacity, construction of specialized cooperative organizations of the main varieties of Chinese herbal medicine in Shandong Province. Through tracing and investigating the four links of planting, processing, circulation and consumption of modern Chinese herbal medicine industry chain, the report explored the general law of the development of Chinese herbal medicine planting industry, analyzed the willingness to grow Chinese herbal medicine, and defined the development status and dynamic trend of Chinese herbal medicine planting industry in Shandong province. Finally, the report find out the problems existing in the development of Chinese herbal medicine planting industry, and put forward targeted solutions. 


\section{Present situation of Chinese herbal medicine planting industry}

Shandong province has the advantages of mild climate, well-developed water system, coupled with four distinct seasons, abundant precipitation and other special resources and environments, so that the production of herbs has a strong comparative advantage of natural resources. In addition to Dezhou, Liaocheng, Binzhou does not have a Chinese herbal medicine planting base, the other 14 city in Shandong province have the habit of planting herbs. There are more than ten kinds of Chinese herbal medicines in Shandong Province, and the main varieties are Sage root, honeysuckle, ginseng, Radix Cynanchi paniculati, Trichosanthes kirilowi etc. In this study, Shandong province was selected as a sample Province, and questionnaire survey and key interviews were conducted among Chinese herb growers in order to know more about the farmers' willingness to grow Chinese herbal medicine and the factors affecting the cultivation of Chinese herbal medicine. This survey was conducted in July to August in 2017 on the basis of the preliminary investigation.

\subsection{Varieties of herbal planting of Shandong Province}

Shandong province is located in the middle latitude region of the northern hemisphere, the eastern part of China, and in the lower course area of the Yellow River. The topography of Shandong province is mostly hilly and plain, and Shandong is rich in soil and developed in water system. The climate in Shandong is temperate monsoon climate with four distinct seasons, rainfall concentrated. All of this is conducive to the cultivation and growth of Chinese herbal medicine. Favorable geographical conditions, coupled with the unique climate and environment, make Shandong province one of the largest provinces of Chinese herbal medicine cultivation. Shandong province has 1470 kinds of Chinese herbal medicine resources, accounting for more than $10 \%$ of Chinese herbal medicine resources. Among them, there are 1299 kinds of botanical herbs, 400 kinds of acquisition of Chinese herbal medicines, 200 kinds of Real estate herbs. There are more than 70 varieties of Chinese herbal medicine planting in Shandong province, and among them, nearly 20 kinds of Chinese herbal medicine cultivation realized a scale-style.

Shandong Province has a long history of Chinese herbal medicine cultivation. The cultivation and processing of Chinese herbal medicine in Shandong province started early and had a good historical accumulation. After the reform and opening up, especially since 1995, Chinese herbal medicine planting industry has been rapid development in Shandong Province, and become a driving force for the adjustment of agricultural industrial structure and promotes the income of the farmers. The total herbal planting area in the province is as high as 1 million 800 thousand mu, accounting for $1 / 10$ of the total planting area of Chinese herbal medicine, and its output value is close to 9 billion Yuan.

\subsection{Present situation of planting scale of Chinese herbal medicine}

With the modernization and the industrial development of Chinese herbal medicine, Chinese herbal medicine planting gradually aroused the concern of the community, and the local governments have also introduced policies to encourage and guide farmers to actively support the Chinese herbal medicine planting industry. Shandong province took advantages of the returning farmland to forest and the agricultural structure strategic adjustment opportunities, strengthened the adjustment of the layout of Chinese herbal medicine production, worked out the development plan of Chinese herbal medicine industry in accordance with local conditions, cleared industry development goal, and gradually established a series of Chinese herbal medicine advantages and characteristics producing areas.

Since the end of last century, Chinese herbal medicine planting areas has extended from Yimeng Mountain, Wendeng, Heze and other traditional areas to the other cities in Shandong province. Currently, in addition to Dezhou, Binzhou, and Liaocheng, the rest of the cities in Shandong have different scale planting areas of Chinese herbal medicine. There are Lin-Ri-Wei-Zi, Ji-Tai-Lai, Wei-Yan-Qin, He-Ji,Dongying 5 large Chinese herbal medicine planting area in Shandong. Among them, Lin-Ri-Wei-Zi is the largest Chinese herbal medicine planting area. Besides, Wendeng 
ginseng, Heze peony, Pingyi honeysuckle, Juxian Huangqi are the regional advantages of Chinese herbal medicine varieties, local planting area accounted for more than $50 \%$ of the province's planting area.

At present, Shandong province takes the Chinese herbal medicine planting industry as one of the pillar industries to deepen the adjustment of agricultural industrial structure, to promote financial growth and increase the income of farmers. Now, there are 10 more-than-10000-mu Chinese herbal medicine planting base, and 6 herbal medicine planting base through the herbal medicine GAP site certification of Chinese. In Shandong, Chinese herbal medicine planting industry is booming.

\subsection{Benefit status of Chinese herbal medicine cultivation}

At present, the development mode of Chinese herbal medicine industry are mostly the form of "cooperatives + base + farmers" or the "company + base + farmers" in Shandong province. The existing Chinese herbal medicine planting cooperatives is more than 200, Chinese herbal medicine sales company (cooperatives) is more than 300, and leading enterprises took "Cultivation Extraction - sales as the main industry chain is more than 20. Chinese herbal medicine industry has been achieved good economic and social benefits.

\section{Comparison of Chinese herbal medicine planting areas in Shandong province}

Chinese herbal medicines are planted according to local conditions. Therefore, different areas of our province planting herbs have significant differences. Only in the real estate areas, Chinese herbal medicine planting industry can be ensured to develop and improve the quality. In recent years, Chinese herbal medicine planting area in our province is becoming more rational layout, the government established Chinese herbal medicine planting base in many real estate areas. And the quality advantage has become increasingly prominent, the yield and quality of medicinal materials are among the best nationwide. On the basis of full understanding of the cultivation of Chinese herbal medicine in Shandong Province in the early stage, We collect relevant Chinese herbal medicine planting data, and selected 4 representative cities to conduct field research in the province.

\subsection{Cultivation of Chinese herbal medicine in Jinan}

Chinese herbal medicine has been planted in Jinan for hundreds of years. As early as the 50s of last century, Chinese herbal medicine was planted in Jinan. But due to many reasons, Chinese herbal medicine planting area in Jinan city has not been very stable, Chinese herbal medicine planting industry has not obtained the very good development. In 2005, the planting area of Chinese herbal medicine in Jinan was only $2400 \mathrm{Mu}$. But in recent years, with the enhancement of people's health consciousness, cultivation of Chinese herbal medicine industry has also been favored by the government. Jinan municipal government formulated the development plan of Chinese herbal medicine planting industry, and put forward "around the bib-cock, relying on the market, developing brands, highlighting features, continuous development" of the Chinese herbal medicine industry development concept. The government encourages the development of Chinese herbal medicine industry.

\subsection{Cultivation of Chinese herbal medicine in Weihai}

Weihai, located in the eastern part of Shandong Peninsula, is a temperate continental monsoon climate. The average annual precipitation is $778.4 \mathrm{~mm}$, with an average annual temperature of 11.5 degrees, with an average annual sunshine duration of 2569.4 hours. Richly endowed by nature geography, climate and environment created Weihai ginseng of higher quality. By the Ministry of agriculture and the product quality supervision and Inspection Center for identification, Weihai American ginseng saponin content as high as 9.2\%, higher than the imported reference 3.6\%. The content of selenium in American ginseng is 7 times higher than that of American ginseng, and the content of harmful heavy metals in Weihai ginseng is up to the national standard. Therefore, Weihai ginseng in the country is renowned. The total annual output of ginseng is 5500 tons, with a total 
output value of up to 1 billion 100 million yuan, which is the largest trading volume of Chinese herbal medicine in Weiha.

\subsection{Cultivation of Chinese herbal medicine in Linyi}

Linyi is the largest Chinese herbal medicine producing area in the province, and is rich in honeysuckle, known as "the hometown of Chinese honeysuckle". Honeysuckle is authentic traditional herbal medicine, has a long history of cultivation in Linyi. Honeysuckle is also one of the most precious herbal medicines in China. It has high medicinal value and the market demand is increasing year by year.At present, honeysuckle planting area is up to 1 million 84 thousand acres in Linyi. Linyi herbal medicine planting area of up to about 1500000 acres, with an annual output of 11 thousand and 360 tons, with a large quantity of Chinese herbal medicine, high quality and competitive advantages, selling well at home and abroad, has become the backbone of promoting local economic development.

\subsection{Cultivation of Chinese herbal medicine in Heze}

Heze city is located in the southwest of Shandong Province, at the junction of the four provinces, located in the lower reaches of the Yellow River, in addition to the Juye hills; the remaining counties are located in the Yellow River alluvial plain, with flat, fertile soil. And the agriculture is well developed. The average annual precipitation is $650 \mathrm{~mm}$, the annual average temperature is 18 degrees, and annual average frost free period is 213 days, which is suitable for the growth of Chinese herbal medicine.

Shun Wangcheng Chinese herbal medicine market is the only national Herb Market in Shandong province. At present, the market covers an area of 140 thousand square meters, construction area of nearly 60 thousand square meters, has the fixed 460 stores, more than 1000 booths, business varieties more than 1100, the annual distribution of various kinds of Chinese herbal medicines more than 4 tons, turnover of 300 million yuan, Some high-quality real estate in materials such as peony, Radix Paeoniae Alba, Radix Isatidis, angelica, safflower, astragalus, rehmannia, Radix trichosanthis, Rhizoma Pinelliae, Platycodon grandiflorum and other home and abroad. More than 20 provinces and cities nationwide merchants gathered here, following the Anhui Bozhou, Hebei Anguo is the national important distribution center for Chinese medicine.

\section{The development countermeasures of Chinese herbal medicine industry in Shandong Province}

\subsection{Increase efforts to Chinese medicine science}

The government should strengthen the technological innovation in the field of Chinese agriculture, and take the construction of Chinese herbal medicine industry technology into a complete system. Pay attention to the research of key technology of production, accelerate science and technology accumulation. Give full play to the guiding role of financial special funds and social capital, and take leading enterprises as the core, and strive for progress in standardization of production technology.

\subsection{Improve the information exchange platform for Chinese herbal medicine planting}

The government should establish a platform for the exchange of information within the industry. According to the release of the Chinese herbal medicine market situation and development prospects forecast information, comprehensive analysis, timely, reasonable planning of different varieties and regional area, give full play to the economic value of Chinese herbal medicine.

\subsection{Maintain stable development of Chinese herbal medicine market}

The government should stabilize the fluctuation of Chinese herbal medicine prices. It is scientific and reasonable to strengthen the state macro control and ensure the pricing system of Chinese herbal medicines in the competent authorities. Try the medicine quasi futures market; choose a large 
amount of Chinese herbal medicines in the implementation of the pilot area. And establish clear futures trading standards and product quality requirements in Chinese herbal medicine market.

\section{Acknowledgement}

This work is partially supported by1. the National Philosophy and Social Science Foundation as an important program, the Assurance Mechanism of Agricultural Product Quality and Safety based on Multi-agent Coordination(No. 15AGL014). It is also partially supported by 2.the Important Research and Development Program of Shandong Province in 2016, Key Issues and System Implementation of Early Warning for Agricultural Product Quality Safety under the Trend of Intelligent Agriculture (No. 2016GSF120013). 3. The Technology System Innovation Plan of Chinese Herbal Medicine Industry of Shandong Province on the 13th Five-Year Plan, The authors would like to acknowledge the referees for their valuable comments and suggestions which helped to improve the presentation of this paper.

\section{Reference}

[1] Zhifen Wang, Ximin Liu. Present situation and Development Countermeasures of Chinese medicinal herbs cultivation in Shandong [J]. Shandong Agricultural Sciences, 2010 177(10)33-36.

[2] Shilin Chen, Jianhe Wei, Jianping Han. Traditional Chinese medicine agriculture and sustainable development of traditional Chinese medicine resources [J]. The world of science and technology modernization of traditional Chinese Medicine, 2007 93(11)26-28.

[3] Faliang Wang. Target requirements and basic countermeasures for sustainable development of Chinese herbal medicine industry [J]. Journal of Taishan, 2007 122(9)46-48. 